\title{
String derivation of two-loop Feynman diagrams*
}

\author{
Lorenzo Magnea ${ }^{\diamond}$ and Rodolfo Russo ${ }^{\ddagger}$ \\ ${ }^{\diamond}$ NORDITA ${ }^{\dagger}$ \\ Blegdamsvej 17, DK-2100 Copenhagen Ø, Denmark \\ $¥$ Dipartimento di Fisica, Politecnico di Torino \\ Corso Duca degli Abruzzi 24, I-10129 Torino, Italy
}

\begin{abstract}
We briefly review the technology involved in extracting the field-theory limit of multiloop bosonic string amplitudes, and we apply it to the evaluation of simple two-loop diagrams involving scalars and gauge bosons.
\end{abstract}

\section{INTRODUCTION}

The discovery of new physics beyond the Standard Model (SM) is most likely to come from experiments performed at the highest available energy; in fact, it is conceivable that hints of such a discovery have already been seen [1]. It is, however, very difficult to substantiate a discovery claim, if the SM predictions on the matter are not sufficiently sharp. A competing explanation of the new phenomena may be constructed within the boundaries of the Standard Model, by tuning or stretching some of its parameters [2]. It is clear that sharpening our techniques to make more precise SM-based predictions on the outcome of high energy experiments is a necessary tool for the discovery of new physics.

Our goal here is to give a progress report on the development of some of these techniques, and we will focus on the calculation of multiloop scattering amplitudes in field theory using string theory as a calculational tool. The method has been in use for several years [3], and recently considerable progress has been made towards its extension to more than one loop [4]. Here we would like to give a flavor of how these calculations are performed, by reviewing some of the general features of the method, and then discussing two simple examples, one in Yang-Mills theory, the second in a scalar field theory.

*) Talk presented at "Beyond the Standard Model V", Balholm, Norway, April 1997. The work described here is part of an ongoing collaboration with P. Di Vecchia, A. Lerda and R. Marotta. Preprint NORDITA-97/57 P.

†) On leave from Università di Torino, Italy. 
By way of introduction, let us briefly recall some of the general features of stringinspired techniques. The field theory limit of a string amplitude is obtained by taking the string tension $T=1 /\left(2 \pi \alpha^{\prime}\right)$ to infinity, decoupling all massive string modes; in this limit, the string world-sheet degenerates into a graph, and the only corners of string moduli space contributing to the field theory result are those where the integrand of the string amplitude exhibits a singular behavior; string moduli are then related to Schwinger proper times in field theory, and string-derived amplitudes are expressed as those derived using the world-line formalism in field theory [5]. The main advantages of resorting to a theory as complex as string theory to perform Feynman diagram calculations are discussed in detail in [3]: concisely, the calculation of a Yang-Mills scattering amplitude is reduced to roughly the size of the calculation of the same amplitude in a scalar theory; loop momentum integrals are already performed, so that helicity methods can be readily employed, and the result for a set of Feynman diagrams of a given topology is presented directly as a Schwinger-parameter integral, bypassing the tensor algebra associated with the propagation of spin-1 particles.

Because of the limited space available, we cannot give details of the derivation of the amplitudes from the string 'master formula'. We will just briefly review the general formalism, and hopefully clarify some aspects of the method by discussing two simple examples.

\section{GENERAL FEATURES OF THE METHOD}

Let us begin by recalling the general expression for the color-ordered $h$-loop $M$-gluon planar amplitude in the open bosonic string [4],

$$
\begin{aligned}
A_{P}^{(h)} & =C_{h} \mathcal{N}_{0}^{M} \int[d m]_{h} \frac{\prod_{i=1}^{M} d z_{i}}{d V_{a b c}} \prod_{i<j}\left[\frac{\exp \left(\mathcal{G}^{(h)}\left(z_{i}, z_{j}\right)\right)}{\sqrt{V_{i}^{\prime}(0) V_{j}^{\prime}(0)}}\right]^{2 \alpha^{\prime} p_{i} \cdot p_{j}} \\
& \times \exp \left[\sum_{i \neq j}\left(\sqrt{2 \alpha^{\prime}} p_{j} \cdot \varepsilon_{i} \partial_{z_{i}} \mathcal{G}^{(h)}\left(z_{i}, z_{j}\right)+\frac{1}{2} \varepsilon_{i} \cdot \varepsilon_{j} \partial_{z_{i}} \partial_{z_{j}} \mathcal{G}^{(h)}\left(z_{i}, z_{j}\right)\right)\right],
\end{aligned}
$$

where only terms linear in each polarization should be kept, and we omitted the color factor $N^{h} \operatorname{Tr}\left(\lambda^{a_{1}} \cdots \lambda^{a_{M}}\right)$. The amplitude for the scattering of $M$ scalars at $h$ loops is obtained by omitting the second line and inserting a factor $\prod_{i}\left[V_{i}^{\prime}(0)\right]^{(-1)}$ in the measure of integration. The fundamental ingredients of Eq. (1) are the bosonic Green function $\mathcal{G}^{(h)}\left(z_{i}, z_{j}\right)$ (the correlator of two scalar fields on the $h$-loop string world sheet), and the measure of integration on moduli space $[d m]_{h}$. Both these quantities depend only on the genus $h$ of the surface. Expressions for the Green function and the measure at two loops, in the field theory limit, will be given below. All geometric quantities are expressed in the Schottky parametrization of Riemann surfaces, which is obtained by cutting and gluing circles on the Riemann sphere. Each pair of circles (each loop) is defined by a projective transformation, 
and parametrized by two fixed points $\eta_{\mu}$ and $\xi_{\mu}$, and by a multiplier $k_{\mu}$, which are related to exponentials of Schwinger proper times in field theory. The projective transformations $V_{i}^{\prime}(z)$ serve to define the amplitude off the mass shell. Finally, a word must be spent on the integration region, which can be deduced by studying the Schottky representation of Riemann surface with boundaries. Let us briefly consider the two-loop case for clarity. First, one uses the overall projective invariance of the Riemann sphere to fix, say, $\xi_{1}, \xi_{2}$ and $\eta_{1}$ to $\infty, 1$ and 0 respectively. The resulting Schottky representation of the string world-sheet is given in Fig. 1.

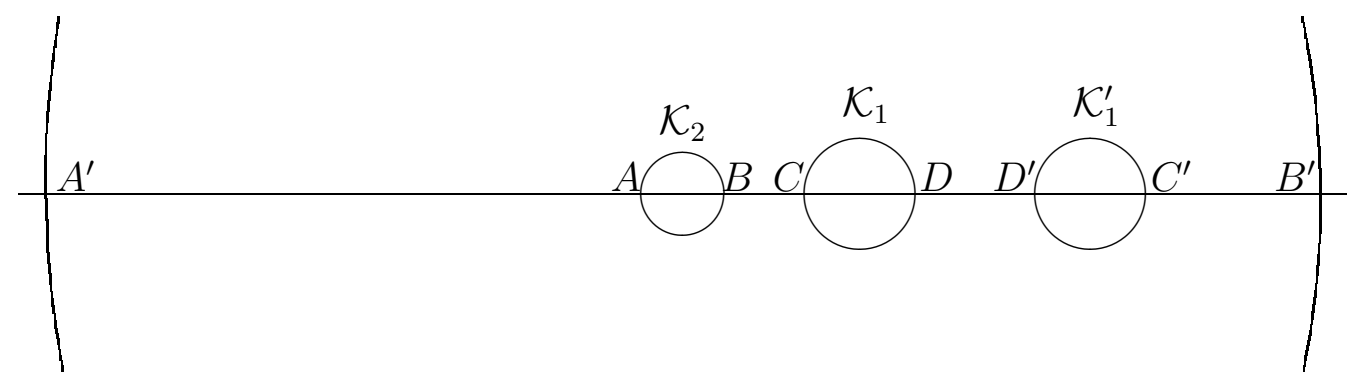

Fig. 1: The two annulus in the Schottky representation.

The two-annulus corresponds to the part of the upper-half plane which is inside the big circle through $A^{\prime}$ and $B^{\prime}$, and outside the other circles; moreover, the points $A, B, C$ and $D$ are identified with $A^{\prime}, B^{\prime}, C^{\prime}$ and $D^{\prime}$ respectively. The segments $\left(A A^{\prime}\right)$ and $\left(D D^{\prime}\right)$ represent the two inner boundaries of the surface, while the union of $(B C)$ and $\left(C^{\prime} B^{\prime}\right)$ represents the external boundary. The positions of these points are simple functions of the parameters $k_{1}, k_{2}$ and $\eta_{2}$ [7]. The region of integration for the multipliers $k_{i}$ and for the remaining fixed point $\eta_{2}$ is determined by the request that the circles do not overlap, and in the field theory limit $\left(k_{i} \rightarrow 0\right)$ it is given by $0 \leq \sqrt{k_{1}} \leq \sqrt{k_{2}} \leq \eta_{2} \leq 1$. The fixed point $\eta_{2}$ can be interpreted as the distance between the two loops, so that one can identify the region $\eta_{2} \rightarrow 1$ as related to reducible diagrams, and the region $\eta_{2} \rightarrow 0$ as related to irreducible ones.

\section{YANG-MILLS VACUUM DIAGRAMS}

To illustrate somewhat more practically the features of Eq. (1), let us consider the case of the two-loop Yang-Mills vacuum bubbles. These are obtained from Eq. (1) by setting $h=2$, omitting all terms involving the Green function (there are no external legs), and expanding the measure of integration to first order in $k_{1}$ and $k_{2}$. In this approximation, Eq. (1) becomes

$$
\begin{aligned}
A_{2}^{0} & =N^{3} C_{2}(2 \pi)^{d} \int \frac{d k_{1}}{k_{1}^{2}} \frac{d k_{2}}{k_{2}^{2}} \frac{d \eta_{2}}{\left(1-\eta_{2}\right)^{2}} \\
& \times\left[1+(d-2)\left(k_{1}+k_{2}\right)+\left((d-2)^{2}+d\left(1-\eta_{2}\right)^{2} \frac{1+\eta_{2}^{2}}{\eta_{2}^{2}}\right) k_{1} k_{2}\right]
\end{aligned}
$$




$$
\begin{aligned}
& \times\left[\ln k_{1} \ln k_{2}-\ln ^{2} \eta_{2}+\frac{2\left(1-\eta_{2}\right)^{2}}{\eta_{2}}\left(k_{1} \ln k_{1}+k_{2} \ln k_{2}\right)+\right. \\
& \left.\quad+\frac{4\left(1-\eta_{2}\right)^{4}}{\eta_{2}^{2}}\left(1+\frac{1+\eta_{2}}{1-\eta_{2}} \ln \eta_{2}\right) k_{1} k_{2}\right]^{-d / 2}
\end{aligned}
$$

There are three singular integration regions that contribute to the amplitude for massless states. The simplest is the region $\eta_{2} \rightarrow 0$, in which the massless states are selected by extracting from the integrand in Eq. (2) the terms proportional to $k_{1}^{-1} k_{2}^{-1} \eta_{2}^{-1}$. Here it is convenient to introduce the variables $q_{1}=k_{2} / \eta_{2}, q_{2}=k_{1} / \eta_{2}$, $q_{3}=\eta_{2}$, which are directly related to the field theory proper times by $t_{i}=-\alpha^{\prime} \ln q_{i}$. The region of integration now takes the form $0 \leq q_{1} \leq q_{2} \leq q_{3} \leq 1$, and the term that survives in the limit $\alpha^{\prime} \rightarrow 0$ is given by

$$
\left.A_{2}^{0}\right|_{q_{3} \rightarrow 0}=\frac{g_{d}^{2}}{(4 \pi)^{d}} N^{3} d(d-2) \int_{0}^{\infty} d t_{2} \int_{0}^{t_{2}} d t_{1} \int_{0}^{t_{1}} d t_{3} \frac{t_{1}+t_{2}+2 t_{3}}{\left(t_{1} t_{2}+t_{1} t_{3}+t_{2} t_{3}\right)^{1+d / 2}}
$$

Notice that the integrand is not symmetric in the exchange of the three proper times, so that the limits of integration cannot be extended to $\infty$ at this stage. This desease is treated by including the second singular region, which can be parametrized as $q_{3}=y q_{2}$. In this region $q_{3}$ and $q_{2}$ go to zero at the same speed (they are not strongly ordered), so that we do not associate any proper time to the variable $y$, which is kept finite. This region contributes

$$
\left.A_{2}^{0}\right|_{q_{3} \rightarrow q_{2}}=-\frac{g_{d}^{2}}{(4 \pi)^{d}} N^{3} 2(d-2) \int_{0}^{\infty} d t_{2} \int_{0}^{t_{2}} d t_{1}\left(2 t_{1} t_{2}+t_{2}^{2}\right)^{-d / 2},
$$

where the integrand can be rewritten as

$$
\left(2 t_{1} t_{2}+t_{2}^{2}\right)^{-d / 2}=\left(t_{1} t_{2}\right)^{-d / 2}+\int_{0}^{t_{1}} d t_{3} \frac{t_{1}+t_{2}}{\left(t_{1} t_{2}+t_{1} t_{3}+t_{2} t_{3}\right)^{1+d / 2}} .
$$

Eq. (4) thus symmetrizes Eq. (3), as well as contributing to a contact interaction.

Finally, in the region $\eta_{2} \rightarrow 1$, we should extract from the integrand of Eq. (2) the terms proportional to $k_{1}^{-1} k_{2}^{-1}\left(1-\eta_{2}\right)^{-1}$, and introduce the proper times $t_{i}=$ $-\alpha^{\prime} \ln k_{i}, t_{3}=-\alpha^{\prime} \ln \left(1-\eta_{2}\right)$. It can be checked that no such terms survive in the limit $\alpha^{\prime} \rightarrow 0$, which is as expected, since the reducible diagram we are considering is zero in Yang-Mills theory. There is however a contact interaction term, leftover from tachyon exchange in the limit $\eta_{2} \rightarrow 1$ (such terms, contributing to diagrams with four-gluon vertices, were present already at one loop [4]). This is obtained by isolating the term in Eq. (2) that is proportional to $k_{1}^{-1} k_{2}^{-1}\left(1-\eta_{2}\right)^{-2}$, and requiring that the integrand be independent of $\eta_{2}$, except for the tachyon double pole. The double pole is then regularized using a $\zeta$-function regularization, amounting to the substitution $\int_{0} d x / x^{2} \rightarrow-1 / 2$. The remaining integral over the proper times $t_{1}$ and $t_{2}$ is then 


$$
\left.A_{2}^{0}\right|_{\eta_{2} \rightarrow 1}=-\frac{g_{d}^{2}}{(4 \pi)^{d}} N^{3} \frac{(d-2)^{2}}{4} \int_{0}^{\infty} d t_{1} d t_{2}\left(t_{1} t_{2}\right)^{-d / 2}
$$

It is easy to check that the sum of Eqs. (6), (3) and (4) correctly reproduces the sum of the corresponding Feynman diagrams in Yang-Mills theory. Notice that the organization of the different terms arising from string theory is very different from what might have been expected in field theory.

\section{A TWO-LOOP SCALAR DIAGRAM}

We will now consider a slightly more complicated diagram, a contribution to the two-point function with both external legs on the same propagator. Since we consider scalar particles (tachyons of the bosonic string), we need the expansions of the various functions in Eq. (1) only to leading order. The measure can be read off from Eq. (2), while the Green function is given by

$$
\begin{aligned}
\mathcal{G}^{(2)}\left(z_{1}, z_{2}\right) & =\log \left(z_{1}-z_{2}\right)+\frac{1}{2}\left[\log k_{1} \log k_{2}-\log ^{2} S\right]^{-1} \\
& \times\left[\log ^{2} T \log k_{2}+\log ^{2} U \log k_{1}-2 \log T \log U \log S\right],
\end{aligned}
$$

where

$$
S=\eta_{2}, \quad T=\frac{z_{2}}{z_{1}}, \quad U=\frac{\left(z_{2}-\eta_{2}\right)\left(z_{1}-1\right)}{\left(z_{1}-\eta_{2}\right)\left(z_{2}-1\right)}
$$

The projective transformations $V_{i}(z)$, to leading order in the multipliers, are given in $[7]$.

Contributions to scalar irreducible diagrams can only come from the region $\eta_{2} \rightarrow$ 0 ; moreover, since we are interested in planar amplitudes, both states must lie on the same boundary of the two-annulus. These observations completely determine the region of integration of the variables in Eq. (1), of which we must now consider only the first line. Furthermore, it is possible to identify the integration region associated with each field theory diagram already at the string level. In the field theory limit, the world-sheet becomes a graph, and each world-sheet boundary degenerates into the union of two distinct propagators, on each of which the external legs may be attached. One can then determine which integration regions for the

punctures $z_{i}$ are related to insertions on the various propagators. For example, if a puncture $z$ lies on the internal boundary represented by the segment $\left(A A^{\prime}\right)$, in the corresponding field theory diagram it will always be attached to the first loop, but will be emitted from the internal propagator if $z \in\left[-1,-\eta_{2}\right]$, or from the external one if $z \in\left[-\eta_{2}, A\right]$ and $z \in\left[A^{\prime},-1\right]$.

In order to show that this identification is correct, let us calculate the two loops diagram with both external states in the region $\left[-1,-\eta_{2}\right]$. Performing the field 
theory limit, we will transform the tachyon into a state of real mass rewriting the quadratic poles $x^{-2} \rightarrow x^{-1-a}=x^{-1} \exp \left[m^{2} \alpha^{\prime} \log x\right]$, as done in Ref. [7].

In the relevant region $\left(-1<<z_{1}<<z_{2}<<-\eta_{2}\right)$ the first line of Eq. (1) becomes

$$
\begin{aligned}
A_{2}^{2} & =\frac{N^{2}}{2^{10}} \frac{g^{4}}{(4 \pi)^{d}} \int_{0}^{\infty} d t_{1} \int_{0}^{t_{1}} d t_{2} \int_{0}^{t_{2}} d t_{3} \mathrm{e}^{-m^{2}\left(t_{1}+t_{2}+t_{3}\right)}\left(t_{1} t_{2}+t_{1} t_{3}+t_{2} t_{3}\right)^{-d / 2} \\
& \times \int_{0}^{t_{3}} d t_{4} \int_{0}^{t_{4}} d t_{5} \exp \left\{p_{1} \cdot p_{2}\left[\left(t_{4}-t_{5}\right)-\frac{\left(t_{1}+t_{2}\right)\left(t_{4}-t_{5}\right)^{2}}{\left(t_{1} t_{2}+t_{1} t_{3}+t_{2} t_{3}\right)}\right]\right\}
\end{aligned}
$$

where new Schwinger parameters $\left(t_{4}=-\alpha \ln \left|z_{1}\right|\right.$ and $\left.t_{5}=-\alpha \ln \left|z_{2}\right|\right)$ have been introduced. The region of integration in Eq. (9) is naturally understandable in a first quantized framework: the proper time related to the punctures cannot exceed the lenght of the propagator on which they are inserted. Identical contributions come from the regions where the order of $z_{1}$ and $z_{2}$ is changed, and where the two external states are attached to the second boundary, represented by the segment $\left(D D^{\prime}\right)$. When the particles are emitted by the two other propagators, one gets contributions that are simply a cyclic permutation of $t_{1}, t_{2}$ and $t_{3}$ in Eq. (9). Taking into account all relevant regions, one gets a completely symmetric expression and can perform the integration over $t_{1}, t_{2}$ and $t_{3}$ independently, introducing a factor of $1 / 3$ !. With the natural change of variables $t_{1,2}=x_{1,2}, t_{3}=x_{3}+x_{4}+x_{5}, t_{4}=x_{4}+x_{5}$ and $t_{5}=x_{5}$, one obtains the final expression

$$
\begin{aligned}
A_{2}^{2} & =\frac{N^{2}}{2^{9}} \frac{g^{4}}{(4 \pi)^{d}} \int_{0}^{\infty} \prod_{i=1}^{5} d x_{i} \Delta^{-d / 2} \mathrm{e}^{-m^{2} \sum_{i=1}^{5} x_{i}} \\
& \times \exp \left\{p_{1} \cdot p_{2}\left[\Delta^{-1}\left(x_{4}\left(x_{1}\left(x_{2}+x_{3}+x_{5}\right)+x_{2}\left(x_{3}+x_{5}\right)\right)\right]\right\},\right.
\end{aligned}
$$

where $\Delta=x_{1} x_{2}+\left(x_{3}+x_{4}+x_{5}\right)\left(x_{1}+x_{2}\right)$. This is the correctly normalized expression for the correspnding diagram in field theory.

Similar calculations with gluons are in progress. For the two-point function, which must be calculated off-shell, the main remaining difficulty is the extension of the expression given in [7] for the projective transformations $V_{i}(z)$ beyond leading order in the multipliers. This difficulty is however irrelevant for higher-point amplitudes, which can be calculated on shell and are independent of the $V_{i}$ 's.

\section{REFERENCES}

1. CDF collaboration, Phys. Rev. Lett. 77 (1996) 438, hep-ex/9601008; H1 collaboration, Z. Phys. C 74 (1997) 191, hep-ex/9702012; ZEUS collaboration, Z. Phys. C 74 (1997) 207, hep-ex/9702015.

2. J. Huston et al. Phys. Rev. D 55 (1997) 1280, hep-ph/9606399; S. Kuhlmann et al., preprint MSU-HEP-70316 and CTEQ-705, hep-ph/9704338.

3. See, for example, M.L. Mangano and S.J. Parke, Phys. Rep. 200 (1991) 301; Z. Bern, L. Dixon and D.A. Kosower, Ann. Rev. Nucl. Part. Sci. 46 (1996) 109, hep-ph/9602280, and references therein. 
4. P. Di Vecchia, A. Lerda, L. Magnea and R. Marotta, Phys. Lett. B 351 (1995) 445, hep-th/9502156. P. Di Vecchia, A. Lerda, L. Magnea, R. Marotta and R. Russo, Nucl. Phys. B 469 (1996) 235, hep-th/9601143; in Erice, Theor. Phys. (1995), hep-th/9602055; L. Magnea and R. Russo, proceedings of the DIS 97 workshop, Chicago, USA (April 1997), hep-th/9706396.

5. C. Schubert, Acta Phys. Polon. B 27 (1996) 3965, hep-th/9610108, and references therein .

6. P. Di Vecchia, F. Pezzella, M. Frau, K. Hornfeck, A. Lerda and S. Sciuto, Nucl. Phys. B 322 (1989) 317.

7. P. Di Vecchia, A. Lerda, L. Magnea, R. Marotta and R. Russo, Phys. Lett. B 388 (1996) 65, hep-th/9607141; K. Roland, Phys. Lett. B 289 (1992) 148. 